\title{
On the parabolic-elliptic limit of the doubly parabolic Keller-Segel system modelling chemotaxis
}

\author{
by \\ Piotr Biler (Wrocław) and Lorenzo Brandolese (Lyon) \\ Dedicated to the memory of Andrzej Hulanicki
}

\begin{abstract}
We establish new results on convergence, in strong topologies, of solutions of the parabolic-parabolic Keller-Segel system in the plane to the corresponding solutions of the parabolic-elliptic model, as a physical parameter goes to zero. Our main tools are suitable space-time estimates, implying the global existence of slowly decaying (in general, nonintegrable) solutions for these models, under a natural smallness assumption.
\end{abstract}

1. Introduction. We consider two related nonlinear parabolic systems which are frequently used as models for chemotactic phenomena, including the aggregation of microorganisms caused by a chemoattractant, i.e. a chemical whose concentration gradient governs the oriented movement of those microorganisms. The parabolic character of the systems comes from the diffusion described by the Laplacians. A version of the system (PE) below is also used in astrophysics as a model of the evolution of a cloud of self-gravitating particles in the mean field approximation.

The first system we consider is the classical parabolic-elliptic Keller-Segel system

$$
\left\{\begin{array}{l}
u_{t}=\Delta u-\nabla \cdot(u \nabla \varphi), \\
\Delta \varphi+u=0, \\
u(0)=u_{0} .
\end{array} \quad x \in \mathbb{R}^{2}, t>0,\right.
$$

Here, $u=u(x, t), \varphi=\varphi(x, t)$ are either functions or suitable (tempered) distributions. When $u \geq 0, \varphi \geq 0$, they may be interpreted as concentrations (densities) of microorganisms and chemicals, respectively.

2000 Mathematics Subject Classification: 35K57, 35B40.

Key words and phrases: Keller-Segel model, chemotaxis, global in time solutions, decay of solutions, blowup of solutions. 
The second system is the parabolic-parabolic system

$$
\left\{\begin{array}{l}
u_{t}=\Delta u-\nabla \cdot(u \nabla \varphi), \\
\tau \varphi_{t}=\Delta \varphi+u, \\
u(0)=u_{0}, \quad \varphi(0)=0,
\end{array} \quad x \in \mathbb{R}^{2}, t>0,\right.
$$

where $\tau>0$ is a fixed parameter. Each of the models can be considered as a single nonlinear parabolic equation for $u$ with a nonlocal (either in $x$ or in $(x, t))$ nonlinearity since the term $\nabla \varphi$ can be expressed as a linear integral operator acting on $u$. In the latter model, the variations of the concentration $\varphi$ are governed by the linear nonhomogeneous heat equation, and therefore are slower than in the former system, where the response of $\varphi$ to the variations of $u$ is instantaneous, and described by the integral operator $(-\Delta)^{-1}$ whose kernel has a singularity. Thus, one may expect that the evolution described by (PE) might be faster than that for (PP), especially for large values of $\tau$ when the diffusion of $\varphi$ is rather slow compared to that of $u$. Moreover, the nonlinear effects for (PE) should manifest themselves faster than for $(\mathrm{PP})$.

The theory of the system (PE) is relatively well developed, in particular when it is studied in a bounded domain in $\mathbb{R}^{d}, d=1,2,3$, with the homogeneous Neumann conditions for $u$ and $\varphi$ at the boundary of the domain. One of the most intriguing properties of $(\mathrm{PE})$ considered for positive and integrable solutions $u$ in the case $d=2$ is the existence of a threshold value $8 \pi$ of mass $M \equiv \int u(x, t) d x$ (see the pioneering work [17] and [1, 3]). Namely, if $u_{0} \geq 0$ is such that $\int u_{0}(x) d x>8 \pi$, then no regular, positive solution $u$ of $(\mathrm{PE})$ can be global in time. For a fine description of the asymptotic behaviour of integrable solutions of (PE) in the subcritical case $M<8 \pi$ we refer the reader to [11] and to [10] for the limit case $M=8 \pi$. See also $[8,9]$ for the radially symmetric case. The higher-dimensional versions of (PE) have also been extensively studied (cf. e.g. [2, 6, 5], and [1] for blow up phenomena).

The doubly parabolic system (PP) has been a bit less studied. For instance, it is known that if for the initial data $u_{0}$ one has $M<8 \pi$, then positive solutions are global in time (see [12] in the case of a bounded planar domain, and also [3, Theorem 5]). However, it is not known whether $M \leq 8 \pi$ is, in general, a necessary condition for the existence of global in time solutions. That is, it is not known whether the blow up occurs for solutions, except for a specific example in [16] of a particular blowing up solution for a system close to (PP). It is even an open question what is the exact range of $M$ guaranteeing the existence of integrable self-similar solutions. For the system (PE) it is proved that $M \in[0,8 \pi)$, and the self-similar solutions (unique for a given $M \in[0,8 \pi)$ ) describe the generic asymptotic behaviour of global in time, positive and integrable solutions of (PE). Concerning (PP), 
it is known that $M<M(\tau)$ with $M(\tau)$ linear in $\tau$ is a necessary condition for the existence of self-similar solutions (cf. [4]). For (PP) with small $M$ such special solutions are also important in the study of space-time decay of general solutions (see [24]). The analysis of whether any $M>8 \pi$ may correspond to a self-similar solution is under way (see [7]). For a different point of view on self-similar solutions for higher-dimensional models of (PP), see also [18]. Usual proofs of blow up for (PE) involve calculations of moments of a solution and then symmetrization (cf. $[11,1])$. These methods do not seem to work for (PP), hence another approach is needed to show blow up for that system. For a numerical insight on blow up issues we refer, e.g., to [14].

A nice result in [25] shows that the solutions of the systems (PP) and (PE) enjoy a kind of stability property as $\tau \searrow 0$ : solutions of (PP) converge in a suitable sense to those of $(\mathrm{PE})$. This had been an old question raised by J. J. L. Velázquez and D. Wrzosek, recently solved in [25]. However, this result, obtained for suitably small solutions in quite a big space of pseudomeasures, gives no indication on the behaviour of possible ("large") blowing up solutions.

The solvability of the systems (PE), (PP) has been studied in various classes of functions and distributions, like Lebesgue, Morrey, Besov, etc., with an immediate motivation to include the a priori strongest possible critical singularities of either solutions or initial data which appear to be point measures in the two-dimensional case and the multiples of the $|x|^{-2}$ function in the higher-dimensional case. In particular, "vast" spaces suitable for analysis of the two-dimensional systems include measure and pseudomeasure spaces (cf. $[2,3,6,25])$.

In this paper we prove the existence of (in general, nonintegrable) solutions in a class $\mathcal{X}$ of functions with natural space-time decay properties (see Theorems 2.1 and 3.1). Here, the space $E$ of admissible initial conditions also contains Dirac measures. The corresponding solutions may be positive and "large" in the sense of being nonintegrable. Nevertheless, they are defined globally in time. Unlike [25], we work in $(x, t)$ space, while [25] has dealt with the Fourier variables $\xi$ (cf. (36) below). Our results are obtained by extension and refinement of techniques used in [5] for (PE) in higher dimensions, but neither for (PP), nor in the two-dimensional case of (PE) which often requires a specific treatement. Moreover, the function spaces we employ allow us to deal with data that can be more singular than those considered in [5]. The spaces $\mathcal{X}$ and $E$ defined in the next section are, in a sense, critical for that analysis, and have already been considered, in slightly different forms, e.g. in the studies of the Navier-Stokes system in [13, 22].

Our main results are contained in Section 4, where we address the problem of convergence as $\tau \searrow 0$, in the space $\mathcal{X}$, of solutions $u^{\tau}$ of (PP) to the 
corresponding solution $u$ of (PE) for small data in $E$. Mathematically, our stability result is not included in, and does not imply, that of [25]. However, it seems that the use of the natural $(x, t)$ variables provides a more immediate physical interpretation. Furthermore, our method looks more flexible, and can be used to prove the stability of the system with respect to stronger topologies. For example, we also establish the convergence in the $L_{t}^{\infty}\left(L_{x}^{1}\right)$ norm for data belonging to $E \cap L^{1}$, and in the $L_{t, x^{\infty}}^{\infty}$ norm for data in $E \cap L^{\infty}$. Motivated by [19], we also address this issue in the more general setting of shift invariant spaces of local measures. The main difficulty in obtaining the convergence $u^{\tau} \rightarrow u$ in strong norms is that $\nabla \varphi^{\tau}$ enjoys some kind of instability as $\tau \rightarrow 0$, in particular in weighted spaces.

Moreover, we give a nonexistence (blow up) result for solutions of (PP) in $\mathbb{R}^{d}, d \geq 1$, with the positive Fourier transform $\widehat{u}_{0}$, in the spirit of [23] (see Theorem 5.1). These are complex-valued solutions with no straightforward physical/biological interpretation. However, such a result tells us that there is no hope to prove the global existence of solutions to (PP) and similar models for arbitrarily large data relying only on size estimates.

2. The parabolic-elliptic system. In order to study the systems (PE) and (PP) we introduce the Banach space $\mathcal{X}$ of functions $u=u(x, t)$ and the Banach space $E$ of tempered distributions $u_{0} \in \mathcal{S}^{\prime}\left(\mathbb{R}^{2}\right)$ by defining the norms

$$
\begin{aligned}
\|u\|_{\mathcal{X}} & =\operatorname{essip}_{t>0, x \in \mathbb{R}^{2}}\left(t+|x|^{2}\right)|u(x, t)|, \\
\left\|u_{0}\right\|_{E} & =\left\|e^{t \Delta} u_{0}\right\|_{\mathcal{X}} .
\end{aligned}
$$

Here, $e^{t \Delta}$ denotes the heat semigroup defined by the Gaussian kernel $g_{t}$, $g_{t}(x)=(4 \pi t)^{-1} e^{-|x|^{2} / 4 t}$. For example, the Dirac mass $u_{0}=\delta$ in $\mathbb{R}^{2}$ is an element of $E$. Notice that, by the definition, $E$ is continuously embedded into the weak Hardy space $\mathcal{H}_{w}^{1}$, which is the space consisting of all tempered distributions $f$ such that $\sup _{t>0}\left|e^{t \Delta} f\right|$ belongs to the Lorentz space $L^{1, \infty}\left(\mathbb{R}^{2}\right)$. See [21].

Let us define the bilinear form $B_{0}$ by

$$
B_{0}(u, v)(t) \equiv \int_{0}^{t} e^{(t-s) \Delta} \nabla \cdot\left(u \nabla(-\Delta)^{-1} v\right)(s) d s .
$$

Here, $(-\Delta)^{-1}$ is the convolution operator on functions defined on $\mathbb{R}^{2}$ with the kernel $K(x)=-(2 \pi)^{-1} \log |x|$. With this notation, the equivalent integral (mild) formulation to (PE), also called the Duhamel formula, reads

$$
u(t)=e^{t \Delta} u_{0}-B_{0}(u, u) .
$$

We begin by establishing the following simple result. 
THEOREM 2.1. There exist two absolute constants $\epsilon, \beta>0$ with the following property. Let $u_{0} \in E$ be such that $\left\|u_{0}\right\|_{E}<\epsilon$. Then there exists a unique (mild) solution $u \in \mathcal{X}$ of $(\mathrm{PE})$ such that $\|u\|_{\mathcal{X}} \leq \epsilon \beta$.

Theorem 2.1 will follow from a series of lemmata.

First, we have the following estimate of the leading term in $\nabla \varphi$.

Lemma 2.1. Let $u \in \mathcal{X}$ and $\varphi$ be such that $\Delta \varphi+u=0$. Then

$$
\nabla \varphi(x, t)=\frac{c_{0} x}{|x|^{2}} \int_{|y| \leq|x| / 2} u(y, t) d y+\mathcal{R}(x, t)
$$

with $c_{0}=-1 / 2 \pi$ and the remainder $\mathcal{R}$ satisfying

$$
|\mathcal{R}(x, t)| \leq C\|u\|_{\mathcal{X}}\left(t^{1 / 2}+|x|\right)^{-1} .
$$

Proof. Indeed, let us represent the partial derivatives of $\varphi$, for $j=1,2$, as

$$
\partial_{j} \varphi=\frac{c_{0} x_{j}}{|x|^{2}} * u \equiv I_{1}+I_{2}+I_{3},
$$

where

$$
I_{1}=\int_{|y| \leq|x| / 2} \frac{c_{0}\left(x_{j}-y_{j}\right)}{|x-y|^{2}} u(y, t) d y .
$$

The terms $I_{2}$ and $I_{3}$ are obtained by taking the integration domains $\{|x-y|$ $\leq|x| / 2\}$ and $\{|x-y| \geq|x| / 2,|y| \geq|x| / 2\}$, respectively. It is straightforward that $I_{2}$ and $I_{3}$ are bounded by $C\|u\|_{\mathcal{X}}\left(t^{1 / 2}+|x|\right)^{-1}$. On the other hand, we can rewrite $I_{1}$ as

$$
\frac{c_{0} x_{j}}{|x|^{2}} \int_{|y| \leq|x| / 2} u(y, t) d y+R_{1}(x, t) .
$$

An application of the Taylor formula shows that the above bound also holds for $R_{1}$.

We immediately deduce from (5) the following useful estimate.

Lemma 2.2. Let $u \in \mathcal{X}$ and $\Delta \varphi+u=0$. Then

$$
\|\nabla \varphi(t)\|_{L^{\infty}} \leq C\|u\|_{\mathcal{X}} t^{-1 / 2}
$$

The last lemma we need is the following.

Lemma 2.3. Let $u, v \in \mathcal{X}$. Then, for some constant $C_{0}$ independent of $u, v$,

$$
\left\|B_{0}(u, v)\right\|_{\mathcal{X}} \leq C_{0}\|u\|_{\mathcal{X}}\|v\|_{\mathcal{X}} .
$$

Proof. We can assume, without any restriction, that $\|u\|_{\mathcal{X}}=\|v\|_{\mathcal{X}}=1$. Lemma 2.2 implies

$$
\left|u \nabla(-\Delta)^{-1} v\right|(x, t) \leq C|x|^{-3 / 2} t^{-3 / 4},
$$


and also

$$
\left|u \nabla(-\Delta)^{-1} v\right|(x, t) \leq C|x|^{-2} t^{-1 / 2}
$$

We denote the gradient of the heat semigroup kernel $g_{t}$ by

$$
G(\cdot, t) \equiv \nabla_{x}\left(\frac{1}{4 \pi t} e^{-|\cdot|^{2} / 4 t}\right)
$$

Then we may represent $B_{0}$ as

$$
B_{0}(u, v)(x, t)=\int_{0}^{t} \int_{0} G(x-y, t-s)\left(u \nabla(-\Delta)^{-1} v\right)(y, s) d y d s \equiv J_{1}+J_{2},
$$

where $J_{1}=\int_{0}^{t} \int_{|y| \leq|x| / 2} \cdots$ and $J_{2}=\int_{0}^{t} \int_{|y| \geq|x| / 2} \cdots$ Using the estimate

$$
|G(x-y, t-s)| \leq C|x-y|^{-5 / 2}(t-s)^{-1 / 4}
$$

and inequality (6a), we get the bound

$$
\left|J_{1}(x, t)\right| \leq C|x|^{-2} .
$$

Another estimate is

$$
\left|J_{1}(x, t)\right| \leq C|x|^{-3 / 2} t^{-1 / 4},
$$

which is obtained using the bound

$$
|G(x-y, t-s)| \leq C|x-y|^{-2}(t-s)^{-1 / 2} .
$$

On the other hand, from the property

$$
\|G(\cdot, t-s)\|_{L^{1}}=c(t-s)^{-1 / 2}
$$

and inequality $(6 \mathrm{~b})$, we obtain

$$
\left|J_{2}(x, t)\right| \leq C|x|^{-2} .
$$

As before, we also have the bound

$$
\left|J_{2}(x, t)\right| \leq C|x|^{-3 / 2} t^{-1 / 4} .
$$

This can be deduced from (6a).

Then, using (8), (11), we obtain the space decay estimate

$$
\left|B_{0}(u, v)\right|(x, t) \leq C|x|^{-2},
$$

and from $(9),(12)$ a (nonoptimal) time decay estimate

$$
\left\|B_{0}(u, v)(t)\right\|_{L^{4 / 3, \infty}} \leq C t^{-1 / 4}
$$

But we may represent $B_{0}$ as

$$
B_{0}(u, v)(t)=e^{t \Delta / 2} B_{0}(u, v)(t / 2)+\int_{t / 2}^{t} G(t-s) *\left(u \nabla(-\Delta)^{-1} v\right)(s) d s
$$


Thus, applying the weak Young-type inequality for convolutions in Lorentz spaces $L^{4 / 3, \infty} * L^{4,1} \subset L^{\infty}$ (see [19]), and the equality obtained from the scaling laws in Lorentz spaces,

$$
\left\|\frac{1}{4 \pi t} e^{-|\cdot|^{2} / 4 t}\right\|_{L^{4,1}}=c t^{-3 / 4}
$$

we finally get

$$
\begin{aligned}
\left\|B_{0}(u, v)(t)\right\|_{L^{\infty}} & \leq C t^{-1}+C \int_{t / 2}^{t}(t-s)^{-1 / 2}\left\|u \nabla(-\Delta)^{-1} v(s)\right\|_{L^{\infty}} d s \\
& \leq C t^{-1}
\end{aligned}
$$

Combining inequalities (13) and (15) we get $B_{0}(u, v) \in \mathcal{X}$, together with its continuity with respect to $u$ and $v$.

Proof of Theorem 2.1. Note that (using the duality $\mathcal{S}-\mathcal{S}^{\prime}$ ) we have $e^{t \Delta} u_{0}$ $\rightarrow u_{0}$ in $\mathcal{S}^{\prime}$ as $t \rightarrow 0$. The conclusion of the theorem follows in a standard way (cf., e.g., $[19,2,6])$ from the contraction fixed point theorem.

3. The parabolic-parabolic system. Let $\tau>0$ be a fixed parameter. We consider the system (PP), whose equivalent integral formulation reads

$$
u(t)=e^{t \Delta} u_{0}-\int_{0}^{t} \nabla \cdot e^{(t-s) \Delta}\left[u(s) \frac{1}{\tau} \nabla \int_{0}^{s} e^{(1 / \tau)(s-\sigma) \Delta} u(\sigma) d \sigma\right] d s .
$$

We introduce for all $\tau \geq 0$ the bilinear form $B_{\tau}$ (recall that $G$ is defined by the expression (7)):

$$
B_{\tau}(u, v)(x, t) \equiv \int_{0}^{t} \int_{0} G(x-y, t-s)\left(u W_{\tau}(v)\right)(y, s) d y d s,
$$

where $W_{\tau}(v)$ is the linear operator acting on $v$ :

$$
W_{\tau}(v)(x, t)=\int_{0}^{t} \frac{1}{\tau}\left[G\left(\frac{t-\sigma}{\tau}\right) * v(\sigma)\right](x, \sigma) d \sigma \quad \text { for } \tau>0,
$$

with a natural convention

$$
W_{0}(v)(x, t)=\left(\nabla(-\Delta)^{-1} v\right)(x, t) .
$$

In this way, the system (PP) is also rewritten in compact form (cf. (4)) as

$$
u=e^{t \Delta} u_{0}-B_{\tau}(u, u) .
$$

We are going to solve (19) in the space $\mathcal{X}$ exactly as in the parabolic-elliptic case. An additional estimate, however, is needed: 
Lemma 3.1. Let $u \in \mathcal{X}$ and $\tau>0$. Then there exists a constant $C^{*}>0$, independent of $u$ and $\tau$, such that

$$
\left\|W_{\tau}(u)(t)\right\|_{L^{\infty}} \leq C^{*} t^{-1 / 2}\|u\|_{\mathcal{X}} .
$$

Proof. As usual, we can and do assume $\|u\|_{\mathcal{X}}=1$. Then for all $1<p \leq \infty$ we have

$$
|u(x, \sigma)| \leq|x|^{-2 / p} \sigma^{-1+1 / p} .
$$

This implies, for $1<p \leq \infty$,

$$
\|u(\sigma)\|_{L^{p, \infty}} \leq \sigma^{-1+1 / p}
$$

Now, we represent

$$
W_{\tau}(u)=I_{1}+I_{2},
$$

where $I_{1}=\int_{0}^{t / 2} \ldots$ and $I_{2}=\int_{t / 2}^{t} \ldots$ Evidently, we obtain the bound

$$
\left\|I_{1}(t)\right\|_{L^{\infty}} \leq C \int_{0}^{t / 2}\left\|\frac{1}{\tau} G\left(\frac{t-\sigma}{\tau}\right)\right\|_{L^{2,1}}\|u(\sigma)\|_{L^{2, \infty}} d \sigma \leq C t^{-1 / 2} .
$$

For the integral $I_{2}$, let us begin with a rough bound

$$
\left\|I_{2}(t)\right\|_{L^{\infty}} \leq C \int_{t / 2}^{t}\left\|\frac{1}{\tau} G\left(\frac{t-\sigma}{\tau}\right)\right\|_{L^{1}}\|u(\sigma)\|_{L^{\infty}} d \sigma \leq C \tau^{-1 / 2} t^{-1 / 2} .
$$

This gives the required estimate, except when $\tau$ belongs to a neighbourhood of the origin. Thus, in what follows, it is enough to consider the case $0<$ $\tau<1 / 2$. Now, we further decompose

$$
I_{2} \equiv I_{2,1}+I_{2,2}
$$

where $I_{2,1}=\int_{t / 2}^{t-\tau t} \ldots$ and $I_{2,2}=\int_{t-\tau t}^{t} \ldots$ Next, we are going to improve (21) by writing

$$
\begin{aligned}
\left\|I_{2,1}(t)\right\|_{L^{\infty}} & \leq C \int_{t / 2}^{t-\tau t}\left\|\frac{1}{\tau} G\left(\frac{t-\sigma}{\tau}\right)\right\|_{L^{3,1}}\|u(\sigma)\|_{L^{3 / 2, \infty}} d \sigma \\
& \leq C \tau^{1 / 6} \int_{t / 2}^{t-\tau t}(t-\sigma)^{-7 / 6} \sigma^{-1 / 3} d \sigma \leq C t^{-1 / 2} .
\end{aligned}
$$

The last estimate is

$$
\begin{aligned}
\left\|I_{2,2}(t)\right\|_{L^{\infty}} & \leq C \int_{t-\tau t}^{t}\left\|\frac{1}{\tau} G\left(\frac{t-\sigma}{\tau}\right)\right\|_{L^{1}}\|u(\sigma)\|_{L^{\infty}} d \sigma \\
& \leq C \tau^{-1 / 2} \int_{t-\tau t}^{t}(t-\sigma)^{-1 / 2} \sigma^{-1} d \sigma \leq C t^{-1 / 2} .
\end{aligned}
$$

The conclusion of Lemma 3.1 follows from (21)-(23). 
Lemma 3.1 allows us to see that if $u, v \in \mathcal{X}$ with $\|u\|_{\mathcal{X}}=\|v\|_{\mathcal{X}}=1$, then

$$
\begin{array}{r}
\left|u W_{\tau}(v)\right|(x, t) \leq C|x|^{-3 / 2} t^{-3 / 4}, \\
\left|u W_{\tau}(v)\right|(x, t) \leq C|x|^{-2} t^{-1 / 2},
\end{array}
$$

for some constant $C>0$ independent of $\tau>0$. These estimates are analogous to those in the parabolic-elliptic case (see (6a) and (6b)). Then, using exactly the same arguments as in the previous section, we arrive at the following existence result.

TheOREM 3.1. There exist two absolute constants $\epsilon^{*}, \beta^{*}>0$ with the following property. Let $u_{0} \in E$ be such that $\left\|u_{0}\right\|_{E}<\epsilon^{*}$. Then there exists a unique (mild) solution $u \in \mathcal{X}$ of $(\mathrm{PP})$ such that $\|u\|_{\mathcal{X}} \leq \epsilon^{*} \beta^{*}$.

REMARK. The case of nonzero initial data $\varphi(0)$ can be studied in a quite similar way.

Remark. A closer look at the proofs of estimates for $\nabla \varphi$ in (PE) and (PP) reveals that the behaviour of $\nabla \varphi$ is a bit different in these two cases. Namely, if $0 \not \equiv u$ and $u(x, t) \sim\left(t+|x|^{2}\right)^{-1}$ (in the sense that $c_{1}\left(t+|x|^{2}\right)^{-1} \leq$ $u(x, t) \leq c_{2}\left(t+|x|^{2}\right)^{-1}$ for some $\left.c_{1}, c_{2}>0\right)$, then it follows from (5) that $\nabla \varphi(x, t) \sim|x|^{-1} \log \left(t+|x|^{2}\right)$ for (PE), while $\nabla \varphi$ is more regular: $|\nabla \varphi(x, t)| \leq$ $c\left(t^{1 / 2}+|x|\right)^{-1}$ in the (PP) case. In other words, letting $\mathcal{Y}$ be the space of functions $f=f(x, t)$ such that $f^{2} \in \mathcal{X}$, we have $\varphi^{\tau} \in \mathcal{Y}$ for $\tau>0$, but $\left(\varphi^{\tau}\right)$ does not converge in $\mathcal{Y}$ as $\tau \rightarrow 0$. However, such an instability does not prevent the convergence of the densities $u^{\tau} \rightarrow u$ for vanishing $\tau$.

4. Study of the $\tau \searrow 0$ limit. We now study the convergence as $\tau \searrow 0$ of solutions $u^{\tau}$ of the system (PP) to the corresponding solution $u$ of (PE). A result in this direction has recently been obtained by A. Raczyński [25], who established the convergence $u^{\tau} \rightarrow u$ in the $\mathcal{Y}_{\alpha}$ norm, for $\alpha \in(1,2)$, defined as

$$
\|u\|_{\mathcal{Y}_{\alpha}}=\operatorname{esssup}_{t>0, \xi \in \mathbb{R}^{2}}\left(1+t^{1 / 2}|\xi|\right)^{\alpha}|\widehat{u}(\xi, t)| .
$$

In Subsection 4.2 we will obtain a similar result using the $\mathcal{X}$-norm.

4.1. Regularity properties of solutions of $(\mathrm{PE})$. In this subsection we prepare some preliminary material. The first proposition concerns regularity of solutions of $(\mathrm{PE})$ with respect to the space variable. The second proposition describes their regularity with respect to the time variable.

Proposition 4.1. For all $r \in(1,2)$ there exists a constant $\epsilon_{r}$ with $0<$ $\epsilon_{r} \leq \epsilon$ (the absolute constant of Theorem 2.1) such that if $\left\|u_{0}\right\|_{E}<\epsilon_{r}$, then 
the solution of $(\mathrm{PE})$ constructed in Theorem 2.1 satisfies

$$
\|\nabla u(t)\|_{L^{r, \infty}} \leq C t^{-3 / 2+1 / r}
$$

for some constant $C=C\left(u_{0}, r\right)$ independent of $t$.

Proof. We use a standard argument involving the subspace

$$
\mathcal{X}_{r}=\left\{u \in \mathcal{X}: \exists C,\|\nabla u(t)\|_{L^{r, \infty}} \leq C t^{-3 / 2+1 / r}\right\},
$$

equipped with its natural norm. Recalling that the kernel $c_{0} x /|x|^{2}$ of the operator $\nabla(-\Delta)^{-1}$ belongs to $L^{2, \infty}$, first we deduce from the Young inequality

$$
\left\|\nabla^{2}(-\Delta)^{-1} v(t)\right\|_{L^{\alpha, \infty}} \leq C t^{-3 / 2+1 / r}\|v\|_{\mathcal{X}_{r}}, \quad \frac{1}{\alpha}=\frac{1}{r}-\frac{1}{2} .
$$

Next, from the Hölder inequality (noticing that $\|u(t)\|_{L^{2, \infty}} \leq C t^{-1 / 2}\|u\|_{\mathcal{X}}$ ),

$$
\left\|u \nabla^{2}(-\Delta)^{-1} v(t)\right\|_{L^{r, \infty}} \leq C t^{-2+1 / r}\|u\|_{\mathcal{X}}\|v\|_{\mathcal{X}_{r}} .
$$

The generalization of the classical inequalities to Lorentz spaces can be found, e.g., in [19].

We claim that the bilinear operator $B_{0}$ introduced in (3) is boundedly defined: $B_{0}: \mathcal{X}_{r} \times \mathcal{X}_{r} \rightarrow \mathcal{X}_{r}$. Indeed, for $\|u\|_{\mathcal{X}_{r}}=\|v\|_{\mathcal{X}_{r}}=1$, we combine the estimate

$$
\|\nabla G(t-s)\|_{1} \leq C(t-s)^{-1}
$$

and (10) with the inequality (a consequence of $u \in \mathcal{X}$ )

$$
\|u(s)\|_{L^{r, \infty}} \leq C s^{-1+1 / r},
$$

the estimate $\left\|\nabla(-\Delta)^{-1} v(s)\right\|_{\infty} \leq C s^{-1 / 2}$ obtained from Lemma 2.2, and (28). Then we arrive at

$$
\begin{aligned}
& \left\|\nabla B_{0}(u, v)(t)\right\|_{L^{r, \infty}} \\
& \leq C \int_{0}^{t / 2}(t-s)^{-1}\|u(s)\|_{L^{r, \infty}}\left\|\nabla(-\Delta)^{-1} v(s)\right\|_{\infty} d s \\
& \quad+C \int_{t / 2}^{t}(t-s)^{-1 / 2}\left(\|\nabla u(s)\|_{L^{r, \infty}}\left\|\nabla(-\Delta)^{-1} v(s)\right\|_{\infty}+s^{-2+1 / r}\right) d s \\
& \leq C t^{-3 / 2+1 / r} .
\end{aligned}
$$

Moreover, for $u_{0} \in E$, we have $\left|e^{(t / 2) \Delta} u_{0}(x)\right| \leq C\left(t+|x|^{2}\right)^{-1}$. Hence, $\left\|e^{(t / 2) \Delta} u_{0}\right\|_{L^{\gamma, \infty}} \leq C t^{-1+1 / \gamma}$ for $1<\gamma<\infty$. We now choose $\beta, \gamma \in(1, \infty)$ such that $1+1 / r=1 / \beta+1 / \gamma$. Then the semigroup property of the heat kernel $g_{t}$, and the fact that $\nabla g_{t / 2} \in L^{\beta, 1}$, imply

$$
\left\|\nabla e^{t \Delta} u_{0}\right\|_{L^{r, \infty}} \leq C t^{-3 / 2+1 / r} .
$$

Now the usual application of the contraction mapping theorem, in a closed ball of small radius in the space $\mathcal{X}_{r}$, allows us to conclude the proof. 
The following proposition is the first crucial tool for our stability result. It provides the Hölder regularity, with respect to the time variable, of solutions of $(\mathrm{PE})$ in Lorentz spaces.

Proposition 4.2. Let $1<r<2$ and $u_{0} \in E$ be such that $\left\|u_{0}\right\|_{E}<\epsilon_{r}$. Then the solution $u$ of $(\mathrm{PE})$ constructed in Proposition 4.1 satisfies, for all $0<t^{\prime}<t$

$$
\left\|u(t)-u\left(t^{\prime}\right)\right\|_{L^{r, \infty}} \leq C\left(t-t^{\prime}\right)^{1 / 2}\left(t^{\prime}\right)^{-3 / 2+1 / r}
$$

for some $C=C\left(u_{0}, r\right)$ independent of $t$ and $t^{\prime}$.

Proof. It is enough to show that both $e^{t \Delta} u_{0}-e^{t^{\prime} \Delta_{u}} u_{0}$ and $B_{0}(u, u)(t)-$ $B_{0}(u, u)\left(t^{\prime}\right)$ satisfy the required bound in the $L^{r, \infty}$-norm.

From the identity

$$
\begin{aligned}
e^{t \Delta} u_{0}(x)-e^{t^{\prime} \Delta} u_{0}(x) & =\int\left(e^{t^{\prime} \Delta} u_{0}(x-y)-e^{t^{\prime} \Delta} u_{0}(x)\right) g_{t-t^{\prime}}(y) d y \\
& =-\iint_{0}^{1} \nabla e^{t^{\prime} \Delta} u_{0}(x-\theta y) \cdot y g_{t-t^{\prime}}(y) d y d \theta
\end{aligned}
$$

we get

$\left\|e^{t \Delta} u_{0}-e^{t^{\prime} \Delta} u_{0}\right\|_{L^{r, \infty}} \leq C\left\|\nabla e^{t^{\prime} \Delta} u_{0}\right\|_{L^{r, \infty}}\left\|y g_{t-t^{\prime}}\right\|_{1} \leq C\left(t-t^{\prime}\right)^{1 / 2}\left(t^{\prime}\right)^{-3 / 2+1 / r}$.

Now, we can write

$$
B_{0}(u, u)(t)-B_{0}(u, u)\left(t^{\prime}\right)=A_{1}+A_{2}
$$

with

$$
\begin{aligned}
& A_{1} \equiv \int_{0}^{t^{\prime}}\left(G(t-s)-G\left(t^{\prime}-s\right)\right) *\left(u \nabla(-\Delta)^{-1} u\right)(s) d s, \\
& A_{2} \equiv \int_{t^{\prime}}^{t} G(t-s) *\left(u \nabla(-\Delta)^{-1} u\right)(s) d s .
\end{aligned}
$$

Recall that from $u \in \mathcal{X}$ we deduce (29). Combining this with the estimate of Lemma 2.2 we get

$$
\left\|u \nabla(-\Delta)^{-1} u(s)\right\|_{L^{r, \infty}} \leq C s^{-3 / 2+1 / r} .
$$

This immediately yields

$$
\left\|A_{2}\right\|_{L^{r, \infty}} \leq \int_{t^{\prime}}^{t}(t-s)^{-1 / 2} s^{-3 / 2+1 / r} d s \leq C\left(t-t^{\prime}\right)^{1 / 2}\left(t^{\prime}\right)^{-3 / 2+1 / r} .
$$

The estimate of $A_{1}$ is slightly more involved. We start with the identity

$$
G(t-s)-G\left(t^{\prime}-s\right)=\left(e^{\left(t-t^{\prime}\right) \Delta}-\mathrm{Id}\right) G\left(t^{\prime}-s\right) .
$$

The action of the convolution operator with the function on the right hand side is studied via the following variant of a result established in [20]. 
Lemma 4.1. Denote the Calderón operator by $\Lambda=(-\Delta)^{1 / 2}$. Then, for some constant $C$ depending only on $r \in(1, \infty)$,

$$
\left\|\left(e^{t \Delta}-\mathrm{Id}\right) f\right\|_{L^{r, \infty}} \leq C t^{1 / 2}\|\Lambda f\|_{L^{r, \infty}} .
$$

Proof. Writing $f=\Lambda^{-1} \Lambda f$, we see that

$$
\left(e^{t \Delta}-\mathrm{Id}\right) f=\Phi_{t} *(\Lambda f),
$$

where

$$
\widehat{\Phi}_{t}(\xi)=t^{1 / 2} \widehat{\Phi}\left(t^{1 / 2} \xi\right) \quad \text { and } \quad \widehat{\Phi}(\xi)=\left(e^{-|\xi|^{2}}-1\right)|\xi|^{-1} .
$$

It only remains to show that $\Phi \in L^{1}\left(\mathbb{R}^{2}\right)$. Indeed, it is well known, and easy to check, that $\widehat{\Psi}(\xi)=|\xi| e^{-|\xi|^{2}}$ defines a function $\Psi \in L^{1}\left(\mathbb{R}^{2}\right)$ (for example, with the method described in [15], one obtains $|\Psi(x)| \leq C(1+|x|)^{-3}$ and $\left.|\nabla \Psi(x)| \leq C(1+|x|)^{-4}\right)$. We conclude by applying the Bochner inequality to the identity

$$
\Phi(x)=-2 \int_{1}^{\infty} \Psi(\eta x) d \eta .
$$

Using this lemma we deduce

$$
\begin{aligned}
\left\|A_{1}\right\|_{L^{r, \infty}} & \leq C\left(t-t^{\prime}\right)^{1 / 2} \int_{0}^{t^{\prime}}\left\|\Lambda \nabla g_{t^{\prime}-s} *\left(u \nabla(-\Delta)^{-1} u\right)(s)\right\|_{L^{r, \infty}} d s \\
& =C\left(t-t^{\prime}\right)^{1 / 2}\left(A_{1,1}+A_{1,2}\right),
\end{aligned}
$$

where $A_{1,1}$ and $A_{1,2}$ are obtained by splitting the integral at $s=t^{\prime} / 2$.

But, as the function $\Psi$ introduced in the proof of Lemma 4.1 satisfies $\nabla \Psi \in L^{1}\left(\mathbb{R}^{2}\right)$, we see by a simple rescaling that

$$
\left\|\Lambda \nabla g_{t^{\prime}-s}\right\|_{1} \leq C\left(t^{\prime}-s\right)^{-1} .
$$

Combining this estimate with inequality (31), we get

$$
A_{1,1} \leq C\left(t^{\prime}\right)^{-3 / 2+1 / r}, \quad 1<r<2 .
$$

For $A_{1,2}$, we combine the estimate

$$
\left\|\Lambda g_{t^{\prime}-s}\right\|_{1} \leq C\left(t^{\prime}-s\right)^{-1 / 2}
$$

with the inequality (for $1<r<2$ )

$$
\left\|(\nabla u)\left(\nabla(-\Delta)^{-1} u\right)(s)\right\|_{L^{r, \infty}}+\left\|u\left(\nabla^{2}(-\Delta)^{-1} u\right)(s)\right\|_{L^{r, \infty}} \leq C s^{-2+1 / r},
$$

obtained by applying (26), Lemma 2.2, and (28) with $u=v$. We get as before

$$
A_{1,2} \leq C\left(t^{\prime}\right)^{-3 / 2+1 / r}, \quad 1<r<2,
$$

and this concludes the proof of Proposition 4.2. 
4.2. The vanishing $\tau$ limit. After Proposition 4.2, the second crucial step for the study of the limit as $\tau \searrow 0$ consists in the asymptotic analysis of the linear operators $W_{\tau}, \tau \geq 0$, introduced in (18a)-(18b). This is the purpose of the following lemma.

LEMmA 4.2. Let $\varepsilon=\varepsilon(\tau)$ be an arbitrary function, strictly increasing and continuous on $[0,1]$, such that $\varepsilon(0)=0$. Let also $1<r<2$ and $u$ be a function satisfying, for $0<t^{\prime}<t$,

$$
\|u(t)\|_{L^{r, \infty}} \leq C t^{-1+1 / r}, \quad\left\|u(t)-u\left(t^{\prime}\right)\right\|_{L^{r, \infty}} \leq C\left(t-t^{\prime}\right)^{1 / 2}\left(t^{\prime}\right)^{-3 / 2+1 / r},
$$

with a constant $C$ independent of $t, t^{\prime}$. Then, for all $t>0, \tau \in[0,1]$, and for another constant $C$, independent of $t$ and $\tau$,

$$
\varepsilon(\tau) \sup _{t>0} t^{1 / 2}\left\|\left(W_{\tau}(u)-W_{0}(u)\right)(t)\right\|_{\infty} \leq C \tau^{1 / r-1 / 2} .
$$

In particular, if $u_{0} \in E$ is small enough (for example, $\left\|u_{0}\right\|_{E} \leq \epsilon_{3 / 2}$ ), then the corresponding solution $u$ of $(\mathrm{PE})$ constructed in Proposition 4.1 satisfies

$$
\lim _{\tau \rightarrow 0} \sup _{t>0} t^{1 / 2}\left\|\left(W_{\tau}(u)-W_{0}(u)\right)(t)\right\|_{\infty}=0 .
$$

Proof. Without any restriction we can assume that $0<\varepsilon(\tau)<1 / 2$ for positive $\tau$. Define $\tilde{\varepsilon}(\tau)$ such that $\varepsilon=\tilde{\varepsilon}^{\frac{1}{r}-\frac{1}{2}}$. Borrowing from [25] the idea of splitting the time integral using intervals depending on $\tau$, we write

$$
W_{\tau}(u)-W_{0}(u) \equiv J_{1}+J_{2}+J_{3},
$$

where

$$
\begin{aligned}
& J_{1}(t)=\int_{0}^{t(1-\tilde{\varepsilon}(\tau))}\left[\frac{1}{\tau} G\left(\frac{t-s}{\tau}\right) * u(s)\right] d s, \\
& J_{2}(t)=\int_{t(1-\tilde{\varepsilon}(\tau))}^{t} \frac{1}{\tau} G\left(\frac{t-s}{\tau}\right) * u(t) d s-W_{0}(u)(t), \\
& J_{3}(t)=\int_{t(1-\tilde{\varepsilon}(\tau))}^{t} \frac{1}{\tau} G\left(\frac{t-s}{\tau}\right) *[u(s)-u(t)] d s .
\end{aligned}
$$

From the first relation of (32) and the Young inequality in Lorentz space (using the fact that, by $(7), G(\cdot, t) \in L^{r^{\prime}, 1}\left(\mathbb{R}^{2}\right)$, where $r^{\prime}$ is the conjugate exponent), we get

$$
\begin{aligned}
\left\|J_{1}(t)\right\|_{\infty} & \leq C \tau^{1 / r-1 / 2} \int_{0}^{t(1-\tilde{\varepsilon}(\tau))}(t-s)^{-1 / 2-1 / r} s^{-1+1 / r} d s \\
& \leq C\left(\frac{\tau}{\tilde{\varepsilon}(\tau)}\right)^{1 / r-1 / 2} t^{-1 / 2} .
\end{aligned}
$$

Notice that this estimate of $J_{1}$ is exactly what we need for (33). 
For $J_{2}$, we see from a simple computation via the Fourier transform that

$$
J_{2}(t)=-g_{t \tilde{\varepsilon}(\tau) / \tau} * W_{0}(u)(t) .
$$

If $1 / \alpha=1 / r-1 / 2$, then we deduce from the usual weak-convolution estimates that $W_{0}(u)=\nabla(-\Delta)^{-1} u$ is bounded in the $L^{\alpha, \infty}$-norm, by $C t^{-1+1 / r}$. Applying once more the Young inequality (using now $g_{t \tilde{\varepsilon}(\tau) / \tau} \in L^{\alpha^{\prime}, 1}$ ), we get, as before,

$$
\left\|J_{2}(t)\right\|_{\infty} \leq C\left(\frac{\tau}{\tilde{\varepsilon}(\tau)}\right)^{1 / r-1 / 2} t^{-1 / 2} .
$$

Applying the second inequality of (32), we obtain immediately

$$
\left\|J_{3}(t)\right\|_{\infty} \leq C \tau^{1 / r-1 / 2} t^{-1 / 2},
$$

which is even better than what we need. This proves (33). Choosing, for example, $r=3 / 2$ and $\varepsilon(\tau)=\tau^{1 / 12} / 2$ proves the last claim (34) of Lemma 4.2.

We are now in a position to establish our first main result.

THEOREM 4.1. There exists an absolute constant $\epsilon^{\prime}>0$ (a priori smaller than the constants $\epsilon, \epsilon^{*}>0$ in Theorems 2.1 and 3.1) such that if $u_{0} \in E$, $\left\|u_{0}\right\|_{E}<\epsilon^{\prime}$, then denoting by $u \in \mathcal{X}$ the solution of $(\mathrm{PE})$ and $u^{\tau} \in \mathcal{X}$ the solution of $(\mathrm{PP})$ constructed in the previous theorems, we have, as $\tau \searrow 0$,

$$
u^{\tau} \rightarrow u \quad \text { in } \mathcal{X} .
$$

Proof. This follows easily from Lemma 4.2. Indeed, from the integral equations (4) and (19), the bilinearity of $B_{\tau}$ and $B_{0}$, and the smallness of the solutions $u^{\tau}$ and $u$, we have (similarly to the reasoning at the beginning of [25, Sec. 5], where two terms in the bilinear expansion can be absorbed by the left hand side)

$$
\left\|u^{\tau}-u\right\|_{\mathcal{X}} \leq C\left\|B_{\tau}(u, u)-B_{0}(u, u)\right\|_{\mathcal{X}} .
$$

But, by the definition of $W_{\tau}$ and $W_{0}$ (see (18a)-(18b)),

$$
B_{\tau}(u, u)(t)-B_{0}(u, u)(t)=\int_{0}^{t} G(t-s) *\left(u\left(W_{\tau}(u)-W_{0}(u)\right)\right)(s) d s .
$$

Arguing as in the proof of Lemma 2.3, we obtain

$$
\left\|B_{\tau}(u, u)-B_{0}(u, u)\right\|_{\mathcal{X}} \leq C\|u\|_{\mathcal{X}}\left(\sup _{t>0} t^{1 / 2}\left\|W_{\tau}(u)-W_{0}(u)(t)\right\|_{\infty}\right) .
$$

If $\epsilon^{\prime}>0$ is small enough, then Lemma 4.2 can be applied to the solution $u$ of $(\mathrm{PE})$, implying that the right hand side of the above inequality has a vanishing limit for small $\tau$. This finally gives

$$
\left\|u^{\tau}-u\right\|_{\mathcal{X}} \rightarrow 0 \quad \text { as } \tau \searrow 0 .
$$

REMARK 4.1. Notice that the smaller the norm $\left\|u_{0}\right\|_{E}$, the faster the convergence $u^{\tau} \rightarrow u$ as $\tau \rightarrow 0$. This is due to the fact that for very small 
data it is possible to apply Lemma 4.2 with $r$ close to 1 (even though the constants in our estimates blow up as $r \searrow 1$ ). More precisely, our arguments show that for any $0<\delta<1 / 2$, one can find a constant $C>0$ and $\epsilon(\delta)>0$ such that, for $\left\|u_{0}\right\|_{E} \leq \epsilon(\delta)$, one has $\left\|u^{\tau}-u\right\|_{\mathcal{X}} \leq C \tau^{1 / 2-\delta}$ for all $0 \leq \tau \leq 1$.

4.3. The parabolic-elliptic limit in stronger topologies. If $u_{0} \geq 0$ is small in the $E$-norm, and belongs to a smaller space, for example, $u_{0} \in E \cap L^{1}$, then the solutions $u^{\tau}$ and $u$ of $(\mathrm{PP})$ and (PE) will remain in $L^{1}$, uniformly in time, during their evolution. Hence, it is natural to ask whether the convergence $u^{\tau} \rightarrow u$ also holds in the natural norm of $L^{\infty}\left((0, \infty) ; L^{1}\right)$. Our next theorem provides a positive answer. As the proof does not really depend on a particular topology, it seems appropriate to consider a more abstract setting.

We denote by $\mathcal{L}$ any shift invariant Banach space of local measures (see [19, Ch. 4] for definition and main properties). These are Banach spaces of distributions, continuously embedded in $\mathcal{D}^{\prime}\left(\mathbb{R}^{2}\right)$. Moreover, they are known to have the following properties (for some constant $C>0$ depending only on $\mathcal{L})$ :

1. For all $f \in \mathcal{L}, g \in L^{1}\left(\mathbb{R}^{2}\right)$, the convolution product $f * g$ is well defined in $\mathcal{L}$ and $\|f * g\|_{\mathcal{L}} \leq C\|f\|_{\mathcal{L}}\|g\|_{1}$.

2. For all $f \in \mathcal{L}, h \in L^{\infty}\left(\mathbb{R}^{2}\right)$, the pointwise product $f h$ is well defined in $\mathcal{L}$ and $\|f h\|_{\mathcal{L}} \leq C\|f\|_{\mathcal{L}}\|h\|_{\infty}$.

3. Each bounded sequence $\left\{f_{k}\right\} \subset \mathcal{L}$ has a subsequence convergent in $\mathcal{L}$, in the distributional sense.

Obvious examples (which are indeed shift invariant spaces of local measures) are the $L^{p}$-spaces, $1<p \leq \infty$, the Lorentz spaces $L^{p, q}, 1<p<\infty, 1<$ $q \leq \infty$, and the space of bounded Borel measures $M\left(\mathbb{R}^{2}\right)=\mathcal{C}_{0}\left(\mathbb{R}^{2}\right)^{*}$. In the last case, duality relations ensure property 3 . Other interesting examples include the Morrey-Campanato spaces $M^{p, q}, 1<p \leq q<\infty$, and suitable multiplier spaces (see [19, Ch. 17]).

On the other hand, the space of pseudomeasures, i.e., of tempered distributions $f$ such that $\widehat{f} \in L^{\infty}$, does not fulfill the second requirement. Therefore, the stability result in the pseudomeasure topology will not be covered by our next theorem, but requires a specific (and more involved) treatment (see [25]).

Notice that, because of the conservation of the total mass for positive solutions of $(\mathrm{PE})$ and $(\mathrm{PP})$, the $L^{1}$-norm remains constant during the evolution. This will allow us to handle the case of data $u_{0} \in E \cap L^{1}$, despite the fact that property 3 breaks down for $L^{1}$.

THEOREM 4.2. Let $u_{0} \in E \cap \mathcal{L}$, where $\mathcal{L}$ is either a shift invariant Banach space of local measures, or $\mathcal{L}=L^{1}$. In the latter case we require either $u_{0} \geq 0$ or, for signed $u_{0}$, that $\left\|u_{0}\right\|_{1}$ is sufficiently small. Then there exists a positive 
constant $\tilde{\epsilon}$, depending only on $\mathcal{L}$, such that if

$$
\left\|u_{0}\right\|_{E}<\tilde{\epsilon}
$$

then the systems $(\mathrm{PE})$ and $(\mathrm{PP})$ have unique solutions $u$ and $u^{\tau}$, respectively, such that for an absolute constant $\tilde{\beta}>0,\|u\|_{\mathcal{X}} \leq \tilde{\beta} \tilde{\epsilon}$ and $\left\|u^{\tau}\right\|_{\mathcal{X}} \leq \tilde{\beta} \tilde{\epsilon}$. In addition,

$$
\sup _{t>0}\|u(t)\|_{\mathcal{L}}<\infty \quad \text { and } \quad \sup _{t>0}\left\|u^{\tau}(t)\right\|_{\mathcal{L}}<\infty .
$$

Moreover, the conclusion $\left\|u^{\tau}(t)-u(t)\right\| \mathcal{X} \rightarrow 0$ of Theorem 4.1 is strengthened to

$$
\sup _{t>0}\left\|u^{\tau}(t)-u(t)\right\|_{\mathcal{L}} \rightarrow 0 \quad \text { as } \tau \searrow 0 .
$$

Proof. Obviously, $\left\|e^{t \Delta} u_{0}\right\|_{\mathcal{L}} \leq C_{0}$ for some constant $C_{0}>0$ independent of $t$. Moreover, for each $\tau \geq 0$ (we thus include the analysis of (PE)), we have the estimate

$$
\left\|B_{\tau}(u, v)(t)\right\|_{\mathcal{L}} \leq \tilde{C}\left(\sup _{t>0}\|u(t)\|_{\mathcal{L}}\right)\|v\|_{\mathcal{X}}
$$

where $\tilde{C}>0$ depends only on $\mathcal{L}$. This follows from (17) written as

$$
B_{\tau}(u, v)(t)=\int_{0}^{t} G(t-s) *\left(u W_{\tau}(v)\right)(s) d s
$$

for each $\tau \geq 0$, with the convention (18b). Therefore, using the usual estimate (10), we have

$$
\left\|B_{\tau}(u, v)(t)\right\|_{\mathcal{L}} \leq C\left(\sup _{t>0}\|u(t)\|_{\mathcal{L}}\right)\left(\sup _{t>0} t^{1 / 2}\left\|W_{\tau}(v)(t)\right\|_{\infty}\right) .
$$

The last factor is bounded by $\|v\|_{\mathcal{X}}$ owing to Lemma 2.2 in the case $\tau=0$, and to Lemma 2.3 for $\tau>0$. This yields (35).

Now, we can consider, for $\tau \geq 0$, the sequence of approximating solutions

$$
u_{k}^{\tau}=e^{t \Delta} u_{0}-B_{\tau}\left(u_{k-1}^{\tau}, u_{k-1}^{\tau}\right), \quad k=1,2, \ldots
$$

When $\tilde{\epsilon}<\min \left\{\epsilon, \epsilon^{*}\right\}$, we know by the proofs of Theorems 2.1 and 3.1 that the sequence $u_{k}^{\tau}$ converges in $\mathcal{X}$ to the solution $u^{\tau}$ of (PE) or (PP). Here, of course, $u=u^{0}$ for the solutions of (PE).

On the other hand, applying recursively $(35)$, we get $u_{k}^{\tau}(t) \in \mathcal{L}$ for all $k$ and

$$
\sup _{t>0}\left\|u_{k}^{\tau}(y)\right\|_{\mathcal{L}} \leq C_{0}+\tilde{\beta} \tilde{C} \tilde{\epsilon}\left(\sup _{t>0}\left\|u_{k-1}^{\tau}(y)\right\|_{\mathcal{L}}\right),
$$

with $\tilde{\beta}=\max \left\{\beta, \beta^{*}\right\}$ (the constants obtained in Theorems 2.1 and 3.1 ). Iterating this inequality we arrive at

$$
\sup _{t>0}\left\|u_{k}^{\tau}(t)\right\|_{\mathcal{L}} \leq C^{\prime}<\infty
$$


provided $\tilde{\beta} \tilde{C} \tilde{\epsilon}<1$. If $\mathcal{L}$ is a shift invariant Banach space of local measures, from property 3 we get, for all $\tau \geq 0$,

$$
\sup _{t>0}\left\|u^{\tau}(t)\right\|_{\mathcal{L}} \leq C^{\prime}<\infty
$$

where $C^{\prime}>0$ is independent of $\tau$. Of course, the last claim remains valid in the case $\mathcal{L}=L^{1}$ and $u_{0} \geq 0$ (notice that the smallness of $\left\|u_{0}\right\|_{E}$ prevents blow up that could occur when the second moment of $u_{0}$ is finite and $\int u_{0}>8 \pi$; see the introduction and the references therein). If we remove the assumption $u_{0} \geq 0$, we can obtain the same conclusion provided $\left\|u_{0}\right\|_{1}$ is sufficiently small. Indeed, we see from inequality (35) that the fixed point argument applies in the space $L_{t}^{\infty}\left(L^{1}\right) \cap \mathcal{X}$.

We now discuss the stability, including also the case $\mathcal{L}=L^{1}$. From the bilinearity of $B_{\tau}$, the mixed estimate (35) and the smallness of the solutions $u$ and $u^{\tau}$ (allowing two terms of the bilinear expansions to be absorbed by the left hand side), we obtain

$$
\left\|u^{\tau}(t)-u(t)\right\|_{\mathcal{L}} \leq C \sup _{t>0}\left\|B_{\tau}(u, u)-B_{0}(u, u)(t)\right\|_{\mathcal{L}}
$$

Arguing as at the end of the proof of Theorem 4.1, we arrive at $\left\|B_{\tau}(u, u)-B_{0}(u, u)(t)\right\|_{\mathcal{L}} \leq C\left(\sup _{t>0}\|u(t)\|_{\mathcal{L}}\right)\left(\sup _{t>0} t^{1 / 2}\left\|\left(W_{\tau}(u)-W_{0}(u)\right)(t)\right\|_{\infty}\right)$, and the conclusion follows from Lemma 4.2 .

REMARK. As an application, observe that taking $\mathcal{L}=L^{\infty}$, we obtain for $u_{0} \in E \cap L^{\infty}$, with $u_{0}$ small in the $E$-norm, $u^{\tau} \rightarrow u$ as $\tau \searrow 0$, uniformly in $(x, t) \in \mathbb{R}^{2} \times[0, \infty)$.

5. Blow up of complex-valued solutions of the parabolic-parabolic system. Consider the system $(\mathrm{PP})$ in the space $\mathbb{R}^{d}$ with any $d \geq 1$. Passing to the Fourier transforms, we may write the Duhamel formula (16) in the form

$$
\begin{aligned}
\widehat{u}(\xi, t)= & e^{-t|\xi|^{2}} \widehat{u}_{0}(\xi) \\
& +\iint_{0}^{t} \int_{0} \frac{\xi \cdot \eta}{\tau} e^{-(t-s)|\xi|^{2}} e^{-(s-\sigma)|\eta|^{2} / \tau} \widehat{u}(\xi-\eta, s) \widehat{u}(\eta, \sigma) d \eta d \sigma d s .
\end{aligned}
$$

Our goal is to construct a class of complex-valued initial data such that the corresponding solutions blow up in finite time, in any classical norm. For $a \in \mathbb{R}$, we denote by $\dot{B}_{\infty}^{a, \infty}$ the homogeneous Besov space, which can also be identified with the Hölder-Zygmund space $\dot{\mathcal{C}}^{a}$. As is well known (see [19, 23]), most of the classical function spaces (including all homogeneous Triebel-Lizorkin, and thus Lebesgue or Sobolev spaces) are continuously embedded in $\dot{B}_{\infty}^{a, \infty}$ for some real $a$. 
THEOREM 5.1. There exists $w_{0} \in \mathcal{S}\left(\mathbb{R}^{d}\right)$ such that $\widehat{w}_{0}(\xi) \geq 0,\left\|\widehat{w}_{0}\right\|_{L^{1}}=1$. If $A$ is sufficiently large, then any (tempered) distributional solution of (36) with $u_{0}=A w_{0}$ (and thus any solution of $(\mathrm{PP})$ ) blows up in finite time. More precisely, for some $t^{*}<\infty$ and each $a \in \mathbb{R}$, we have $\left\|u\left(t^{*}\right)\right\|_{\dot{B}_{\infty}^{a, \infty}}=\infty$.

Our approach is close to that in [5, Theorem 3.1], which followed the argument in [23] for the "cheap" Navier-Stokes equations. We produce some estimates from below of the Fourier transform of any solution with $u(0)=u_{0}$ that can be obtained via the iteration procedure for (36) with

$$
u_{k+1}=e^{t \Delta} u_{0}-\int_{0}^{t} \nabla \cdot e^{(t-s) \Delta} \frac{1}{\tau}\left[u_{k}(s) \nabla \int_{0}^{s} e^{(1 / \tau)(s-\sigma) \Delta} u_{k}(\sigma) d \sigma\right] d s
$$

for $k=1,2, \ldots$ This recurrence relation, in general, does not preserve the positivity of the Fourier transform of $u_{0}$. This leads us to restrict our attention to data of the form $\widehat{u}_{0}=A \widehat{w}_{0}$ with $w_{0} \in \mathcal{S}\left(\mathbb{R}^{d}\right)$ such that supp $\widehat{w}_{0} \subset$ $\left\{\xi \in \mathbb{R}^{d}: 2^{-1} \leq \xi_{1} \leq|\xi| \leq 1\right\}$. For $k=0,1, \ldots$ define

$$
E_{k}=\left\{\xi \in \mathbb{R}^{d}: 2^{k-1} \leq \xi_{1} \leq|\xi| \leq 2^{k}\right\} .
$$

Then for $w_{k}=w_{0}^{2^{k}}$, we have $\widehat{w}_{k}=(2 \pi)^{-d} \widehat{w}_{k-1} * \widehat{w}_{k-1}$, and therefore $\operatorname{supp} \widehat{w}_{k} \subset E_{k}$. This implies that if, in addition, $\widehat{w}_{0}(\xi) \geq 0$, then the positivity of the Fourier transform will be preserved by the sequence $u_{k}$, and so by $u$. The next lemma tells us more:

Lemma 5.1. For all $k=0,1,2, \ldots$, we have

$$
\widehat{u}(\xi, t) \geq \beta_{k} e^{-2^{k} t} \mathbf{1}_{t_{k} \leq t<t^{*}}(t) \widehat{w}_{k}(\xi),
$$

where $\left\{t_{k}\right\}$ and $\left\{\beta_{k}\right\}$ are defined below in (38) and (39).

Proof. For $k=0$, the conclusion immediately follows from

$$
\widehat{u}(\xi, t) \geq A e^{-t|\xi|^{2}} \widehat{w}_{0}(\xi),
$$

provided we choose $\beta_{0}=A$ and $t_{0}=0$. Let $k \geq 1$. Assume that (37) holds for $k-1$. Then, for all $t_{k} \leq t<t^{*}$,

$$
\begin{array}{r}
\widehat{u}(\xi, t) \geq \int_{t_{k-1}}^{t} \int_{t_{k-1}}^{s} \int_{\mathbb{R}^{d}} \frac{\xi_{1} \eta_{1}}{\tau} e^{-(t-s)|\xi|^{2}} e^{-(s-\sigma)|\eta|^{2} / \tau} \beta_{k-1}^{2} e^{-2^{k-1} s} e^{-2^{k-1} \sigma} \\
\times \frac{\widehat{w}_{k-1}(\xi-\eta) \widehat{w}_{k-1}(\eta)}{(2 \pi)^{d}} d \eta d \sigma d s \\
\geq \int_{t_{k-1}}^{t} \int_{\mathbb{R}^{d}}\left(s-t_{k-1}\right) \frac{\xi_{1} 2^{k-2}}{\tau} e^{-(t-s)|\xi|^{2}} e^{-\left(t^{*}-t_{k-1}\right) 2^{2 k-2} / \tau} \cdot \beta_{k-1}^{2} e^{-2^{k} s} \\
\times \frac{\widehat{w}_{k-1}(\xi-\eta) \widehat{w}_{k-1}(\eta)}{(2 \pi)^{d}} d \eta d s .
\end{array}
$$


Thus, we can bound $\widehat{u}(\xi, t)$ from below as follows:

$$
\begin{aligned}
& \widehat{u}(\xi, t) \\
& \geq \int_{t_{k-1}}^{t}\left(s-t_{k-1}\right) \frac{2^{2 k-3}}{\tau} e^{-(t-s) 2^{2 k}} e^{-\left(t^{*}-t_{k-1}\right) 2^{2 k-2} / \tau} \beta_{k-1}^{2} e^{-2^{k} s} \widehat{w}_{k}(\xi) d s \\
& \geq\left(\int_{t_{k-1}}^{t}\left(s-t_{k-1}\right) e^{-(t-s) 2^{2 k}} d s\right) \frac{2^{2 k-3}}{\tau} e^{-\left(t^{*}-t_{k-1}\right) 2^{2 k-2} / \tau} \beta_{k-1}^{2} e^{-2^{k} t} \widehat{w}_{k}(\xi) \\
& =\left(\frac{t-t_{k-1}}{2^{2 k}}-\frac{1-e^{-\left(t-t_{k-1}\right) 2^{2 k}}}{2^{4 k}}\right) \frac{2^{2 k-3}}{\tau} e^{-\left(t^{*}-t_{k-1}\right) 2^{2 k-2} / \tau} \beta_{k-1}^{2} e^{-2^{k} t} \widehat{w}_{k}(\xi) \\
& \geq\left(\left(t_{k}-t_{k-1}\right)-\frac{1-e^{-\left(t^{*}-t_{k-1}\right) 2^{2 k}}}{2^{2 k}}\right) \frac{2^{-3}}{\tau} e^{-\left(t^{*}-t_{k-1}\right) 2^{2 k-2} / \tau} \beta_{k-1}^{2} e^{-2^{k} t} \widehat{w}_{k}(\xi)
\end{aligned}
$$

This suggests setting, for some $\delta>0, t^{*}-t_{k-1}=\delta \tau 2^{-2 k+2}$. With this choice, putting $t_{0}=0$, we have

$$
t^{*}=\delta \tau, \quad t_{k}=\delta \tau\left(1-2^{-2 k}\right) .
$$

Then $t_{k}-t_{k-1}=3 \delta \tau 2^{-2 k}$. We get, for $t_{k} \leq t<t^{*}$,

$$
\widehat{u}(\xi, t) \geq\left(3 \delta \tau-1+e^{-4 \delta \tau}\right) 2^{-2 k-3} \tau^{-1} e^{-\delta} \beta_{k-1}^{2} e^{-2^{k} t} \widehat{w}_{k}(\xi) .
$$

This inequality is interesting only if the right hand side is positive. Therefore we will assume that $3 \delta \tau \geq 1$. We choose $\left\{\beta_{k}\right\}$ in such a way that

$$
\beta_{0}=A, \quad \beta_{k}=\left(3 \delta \tau-1+e^{-4 \delta \tau}\right) 2^{-2 k-3} \tau^{-1} e^{-\delta} \beta_{k-1}^{2}, \quad k=1,2, \ldots
$$

This choice leads to inequality (37).

In order to compute $\beta_{k}$, we introduce $M_{\delta, \tau}$ such that

$$
2^{M_{\delta, \tau}}=\left(3 \delta \tau-1+e^{-4 \delta \tau}\right) e^{-\delta} 2^{-3} \tau^{-1} .
$$

Notice that $\beta_{k}=2^{M_{\delta, \tau}-2 k} \beta_{k-1}^{2}$. We claim that, for some $a, b, c \in \mathbb{R}$,

$$
\beta_{k}=A^{2^{k}} 2^{a+b k+c 2^{k}} .
$$

Indeed, from an easy calculation we find $b=2, a=4-M_{\delta, \tau}$ and finally $c=M_{\delta, \tau}-4$, which is needed to ensure $\beta_{0}=A$. Hence,

$$
\beta_{k}=\left(A 2^{M_{\delta, \tau}-4}\right)^{2^{k}} 2^{4-M_{\delta, \tau}+2 k}, \quad k=0,1, \ldots
$$

We conclude that if

$$
A \geq 2^{4-M_{\delta, \tau}},
$$

then by (39) we have $\beta_{k} \rightarrow \infty$ and, in particular, $\left\|u\left(t_{k}\right)\right\|_{L^{1}}=\left\|\widehat{u}\left(t_{k}\right)\right\|_{\infty} \rightarrow \infty$ for $k \rightarrow \infty$. The above size condition on $A$ can be rewritten equivalently as

$$
\left(3 \delta \tau-1+e^{-4 \delta \tau}\right) A \geq 2^{7} e^{\delta} \tau .
$$


A further analysis of the lower bounds obtained for the Fourier transform of a candidate solution permits us to conclude, as in [5], that $\left\|u\left(t^{*}\right)\right\|_{\dot{B}_{\infty}^{a, \infty}}=\infty$ for each $a \in \mathbb{R}$, so that all Besov (and also $L^{p}$ or Triebel-Lizorkin) norms of $u$ blow up not later than at $t^{*}$. Notice that, for a blow up at $t^{*}=1$, we need $A \geq C e^{1 / \tau} \tau$ (cf. (38)).

Acknowledgments. This research has been supported by the European Commission Marie Curie Host Fellowship for the Transfer of Knowledge "Harmonic Analysis, Nonlinear Analysis and Probability" MTKD-CT-2004013389, and by the Polish Ministry of Science (MNSzW) grant-project N201 022 32/0902.

\section{References}

[1] P. Biler, Existence and nonexistence of solutions for a model of gravitational interaction of particles, III, Colloq. Math. 68 (1995), 229-239.

[2] - , The Cauchy problem and self-similar solutions for a nonlinear parabolic equation, Studia Math. 114 (1995), 181-205.

[3] —, Local and global solvability of parabolic systems modelling chemotaxis, Adv. Math. Sci. Appl. 8 (1998), 715-743.

[4] -, A note on the paper of Y. Naito, in: Self-Similar Solutions of Nonlinear PDE, Banach Center Publ. 74, Inst. Math., Polish Acad. Sci., Warszawa, 2006, 33-40.

[5] P. Biler and L. Brandolese, Global existence versus blow up for some models of interacting particles, Colloq. Math. 106 (2006), 293-303.

[6] P. Biler, M. Cannone, I. Guerra and G. Karch, Global regular and singular solutions for a model of gravitating particles, Math. Ann. 330 (2004), 693-708.

[7] P. Biler, L. Corrias and J. Dolbeault, in preparation.

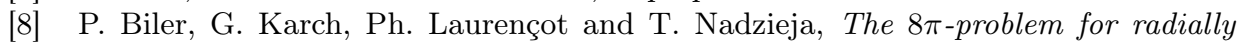
symmetric solutions of a chemotaxis model in a disc, Topol. Methods Nonlinear Anal. 27 (2006), 133-144.

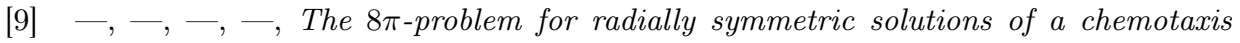
model in the plane, Math. Methods Appl. Sci. 29 (2006), 1563-1583.

[10] A. Blanchet, J. A. Carrillo and N. Masmoudi, Infinite time aggregation for the critical Patlak-Keller-Segel model in $\mathbb{R}^{2}$, Comm. Pure Appl. Math. 61 (2007), 14491481.

[11] A. Blanchet, J. Dolbeault and B. Perthame, Two-dimensional Keller-Segel model: Optimal critical mass and qualitative properties of the solutions, Electron. J. Differential Equations 2006, no. 44, 33 pp.

[12] V. Calvez and L. Corrias, The parabolic-parabolic Keller-Segel model in $\mathbb{R}^{2}$, Comm. Math. Sci. 6 (2008), 417-447.

[13] T. Cazenave, F. Dickstein and F. Weissler, Chaotic behavior of solutions of the Navier-Stokes system in $\mathbb{R}^{N}$, Adv. Differential Equations 10 (2005), 361-398.

[14] F. Filbet, A finite volume scheme for the Patlak-Keller-Segel chemotaxis model, Numer. Math. 104 (2006), 457-488.

[15] G. Furioli and E. Terraneo, Molecules of the Hardy space and the Navier-Stokes equations, Funkcial. Ekvac. 45 (2002), 141-160.

[16] M. Herrero and J. J. L. Velázquez, A blow-up mechanism for a chemotaxis model, Ann. Scuola Norm. Sup. Pisa Cl. Sci. (4) 24 (1997), 633-683. 
[17] W. Jäger and S. Luckhaus, On explosions of solutions to a system of partial differential equations modelling chemotaxis, Trans. Amer. Math. Soc. 329 (1992), 819-824.

[18] H. Kozono and Y. Sugiyama, Keller-Segel system of parabolic-parabolic type with initial data in weak $L^{n / 2}\left(\mathbb{R}^{n}\right)$ and its applications to self-similar solutions, Indiana Univ. Math. J. 57 (2008), 1467-1500.

[19] P.-G. Lemarié-Rieusset, Recent Developments in the Navier-Stokes Problem, Chapman \& Hall/CRC Press, Boca Raton, FL, 2002.

[20] Y. Meyer, Wavelets, paraproducts and Navier-Stokes equations, in: Current Developments in Mathematics, Int. Press, Cambridge, MA, 1997, 105-212.

[21] T. Miyakawa, Application of Hardy space techniques to the time-decay problem for incompressible Navier-Stokes flow in $\mathbb{R}^{n}$, Funkcial. Ekvac. 41 (1998), 383-434.

[22] - On space-time decay properties of nonstationary incompressible Navier-Stokes flows in $\mathbb{R}^{n}$, ibid. 43 (2000), 541-557.

[23] S. Montgomery-Smith, Finite time blow up for a Navier-Stokes like equation, Proc. Amer. Math. Soc. 129 (2001), 3025-3029.

[24] Y. Naito, Asymptotically self-similar solutions for the parabolic system modelling chemotaxis, in: Self-Similar Solutions of Nonlinear PDE, Banach Center Publ. 74, Inst. Math., Polish Acad. Sci., Warszawa, 2006, 149-160.

[25] A. Raczyński, Stability property of the two-dimensional Keller-Segel model, Asymptot. Anal. 61 (2009), 35-59.

Instytut Matematyczny

Uniwersytet Wrocławski

pl. Grunwaldzki $2 / 4$

50-384 Wrocław, Poland

E-mail: Piotr.Biler@math.uni.wroc.pl
Université de Lyon

Université Lyon 1

Institut Camille Jordan, CNRS UMR 5208

43 bd. du 11 Novembre

69622 Villeurbanne Cedex, France

E-mail: brandolese@math.univ-lyon1.fr

Received April 7, 2008

Revised version March 9, 2009 